\title{
Piggyback Technique with and without Inferior Vena Cava Cross- Clamping for Orthotopic Liver Transplant
}

\author{
Marcelo José Antunes Sette ${ }^{1, *}$, Edmundo Pessoa de Almeida Lopes², Álvaro Antônio Bandeira \\ Ferraz $^{1}$, Marcelo Maia ${ }^{3}$, Mauricio Fernando Almeida Barros ${ }^{4}$, Marcel Cerqueira Cesar Machado ${ }^{4}$, \\ Telesforo Bacchella ${ }^{4}$, Renato Cury ${ }^{4}$, Hoel Sette Jr. ${ }^{4}$ and Edmundo Machado Ferraz ${ }^{5}$
}

\begin{abstract}
${ }^{I}$ The Liver Transplant Team at the Memorial São José Hospital, and the Dept. of Surgery, Federal University of Pernambuco, Brazil. Rua Jader de Andrade, 227 - Recife, PE 52061-060, Brazil; ${ }^{2}$ The Liver Transplant Team at the Memorial São José Hospital, and the Dept. of Internal Medicine, Federal University of Pernambuco, Brazil; ${ }^{3}$ The Liver Transplant Team at the Memorial São José Hospital, Brazil; ${ }^{4}$ The Liver Transplant Team of the Dept. of Gastroenterology, University of São Paulo, Brazil and ${ }^{5}$ Dept. of Surgery, Federal University of Pernambuco, Brazil
\end{abstract}

\begin{abstract}
This study aimed to compare the piggyback technique with and without inferior vena cava cross-clamping (IVC-CC). Between 2002 and 2005 at two Hospitals in Brazil, 136 patients were submitted to orthotopic liver transplant (OLT), but 36 were excluded due to the employment of different techniques. Depending on the piggyback technique employed, the remaining 100 patients were divided into two groups: Group A (with IVC-CC) = 47 patients; and Group B (without IVC-CC) $=53$ patients. The study revealed that the OLT using piggyback with IVC-CC took less time (1.39 hours) and required less blood transfusion, however a higher dosis of noradrenaline administration was necessary. No statistical differences were observed between the two groups regarding hemodynamic parameters during the surgery, or any impairment of the kidney and liver functions in the early post-operative period. In conclusion, the piggyback with IVC$\mathrm{CC}$ required less surgical time and less units of blood transfusion.
\end{abstract}

Key Words: Piggyback, liver transplant, inferior vena cava.

\section{INTRODUCTION}

The piggyback technique has been one of the most important advances in orthotopic liver transplant (OLT) surgery over the last decades. First described by Calne and Williams, and later widely divulged by Tzakis et al., this new technique improved hepatectomy with inferior vena cava (IVC) preservation [1-3]. By preserving the IVC blood flow, any harmful effects caused by venous hypertension, venous stasis are prevented. Although this technique has been used in most liver Centers since the late 1980's, it is nonetheless inadvisable to employ this technique in certain circumstances such as liver hypertrophy. In these cases the hypertrophic caudate lobe significantly involves the IVC retrohepatic portion, causing its removal to be practically mandatory during hepatectomy [4].

Originally, the piggyback technique with inferior vena cava cross-clamping (IVC-CC) and liver removal with recipient IVC preservation was performed using a venovenous bypass involving the IVC, portal vein and right axillary vein. Here, IVC-CC was carried out to prevent bleeding during the dissection of the retrocaval area as well as to lead to a safer supra-hepatic vena cava anastomosis, as the surgeon has much more space [1,3]. Later, Belghiti et al. proposed a modification of this technique, which employed neither IVC-CC nor

*Address correspondence to this author at the Rua Jader de Andrade, 227, 52061-060 - Recife, PE, Brazil; E-mail: marcelosette@globo.com venovenous bypass $[5,6]$. Thus, IVC lockage was avoided, the deleterious effects of venous hypertension and venous stasis in the IVC territory could be prevented, as well as systemic arterial hypotension resulting from reduced venous return.

At two General Hospitals in Brazil the piggyback technique for OLT is employed both with IVC-CC as often as without IVC-CC. In neither of these cases a venovenous bypass is employed. This study has aimed to compare the two variants of the piggyback technique - with and without IVC$\mathrm{CC}$ - by means of a retrospective analysis of the following parameters: duration of intervention, packed red cell needs, hemodynamic intraoperative changes, and immediate $(48 \mathrm{~h})$ postoperative kidney and liver dysfunctions.

\section{PATIENTS AND METHODS}

All 136 patients undergoing OLT from Jan/2002 to Aug/2005 at two General Hospitals, one in Recife and another in Sao Paulo, Brazil, were retrospectively evaluated. Due to the employment of techniques other than those proposed by this study, 36 patients were excluded: 12 patients underwent conventional OLT, 11 living-related liver transplantation, 8 were submitted to retransplantation, 4 had domino liver transplant, and 1 a split-liver. Thus, the 100 included patients were submitted to the same intervention and anesthetic protocol, and grouped according to the selected transplant technique: 
- Group A: 47 patients, piggyback technique with IVC$\mathrm{CC}$;

- Group B: 53 patients, piggyback technique without IVCCC.

\section{Surgical Technique}

After entering the abdomen, the liver was identified and the vascular hilum elements dissected, followed by ligation of every structure but the portal vein. Next, the IVC was dissected free, and the hepatectomy itself was carried out through the ligation and division of the portal vein. In the piggyback technique with IVC-CC, the IVC was exposed, supra and infra-hepatic vascular clamps were applied, with the IVC being dissected free from the liver up to the outlet of the hepatic veins. Finally, the three hepatic veins were prepared to form a joined ostium for anastomosis with the graft suprahepatic IVC. In the piggyback technique without IVC$\mathrm{CC}$, the venous ligations between the IVC and the caudate lobe were carried out one by one until the hepatic veins became exposed [2]. Following this, a vascular clamp was placed in a manner that totally obstructed the hepatic vein and partially occluded the IVC. Only at this point did the hepatectomy take place [2].

Selection of the surgical technique was left to the criteria of the surgeon designated for that day's procedure by a previously prepared duty schedule. For example, on Thursday the surgeon usually makes the procedure with IVC-CC; on the other hand on Saturday the surgeon use the technique without IVC-CC, becoming the surgical option aleatory. Four senior-surgeons were involved in this study. According to their better experience, two of them always did the operation with IVC-CC technique and the others without IVC-CC. All of them have approximately 20 years of experience in the liver transplantation field and around 10 years making the piggyback technique with and without IVC-CC.

Surgery time was recorded from the moment of incision to closure of the abdominal wall. For analysis, the intervention was divided in three periods. Step I period refers to the initial stages of anesthesia just after opening the abdominal cavity; Step II encompasses the anhepatic period immediately after native liver removal until the graft revascularization; and Step III is the period immediately prior to abdominal wall closure until the patient was discharged from the operating room. Blood loss was estimated through the amount of packed red cells (PRC) supplied during the intraoperative period.

\section{Hemodynamic Data}

The hemodynamic study was performed through the assessment of the mean arterial pressure (MAP), by means of left radial artery puncture, checked by a membrane transducer, and expressed in mmHg. Heart rate (HR) was recovered from the cardiac monitor, and the central venous pressure (CVP) was measured from a Swan-Ganz catheter connected to a membrane transducer and expressed in $\mathrm{mmHg}$. Three readings were made for each parameter analyzed, and the final number represents the mean value of the three previously registered values.

The amount of fluids infusion, fresh frozen plasma (FFP), noradrenaline administration and diuresis from the moment of incision until the closure of the abdominal wall were also measured.

\section{Blood Laboratory Data}

Blood samples were taken preoperatively at hospital admission for OLT, and on the second post-operative day in order to measure the serum levels of aspartate aminotransferase (AST), alanine aminotransferase (ALT), total bilirubin (TB), blood urea nitrogen (BUN), creatinine $(\mathrm{Cr})$, and the international normalized ratio (INR). The MELD score (Model for End-Stage Liver Disease) was calculated from the results at admission.

\section{Statistical Analysis}

The statistical analysis was carried out using the SigmaStat Software for Windows 3.10, Copyright 2004 Systat software. The frequency analysis was conducted with the chisquare test; and the inter-group comparisons were performed with the multivariate analysis (ANOVA). A significance level of $p<0.05$ was chosen.

\section{RESULTS}

When comparing age, gender, $\mathrm{ABO}$ blood groups, and MELD scores for Groups $\mathrm{A}$ and $\mathrm{B}$, a matching was observed, with no significant difference being detected between the two groups. The mean age of patients from Groups A and B were $47,34 \pm 14,01$ years and $42,77 \pm 14,40$ years, respectively. The MELD score from Group A was 20,55 $\pm 10,45$ and $B$ was 23,69 $\pm 9,94$. In addition, the etiology of the liver disease resulting in OLT for all 100 patients evaluated revealed no statistical difference in either group (Table 1). Early mortality until the second postoperative day was $17 \%$ (8 out of 47) for Group A, and 32\% (17 out of 53), for Group $\mathrm{B}(\mathrm{p}=1.0)$. The major mortality in this period was primary-

Table 1. Etiology of the Liver Diseases in 100 Patients Submitted to Orthotopic Liver Transplant Performed by Piggyback Technique with (Group $A)$ and without (Group B) Inferior Vena Cava Cross-Clamping*

\begin{tabular}{|l|c|c|c|}
\hline \multicolumn{1}{|c|}{ Etiology } & $\begin{array}{c}\text { Group A } \\
\text { N (\%) }\end{array}$ & $\begin{array}{c}\text { Group B } \\
\text { N (\%) }\end{array}$ & P \\
\hline \hline Hepatitis C virus (HCV) & $13(27.6)$ & $13(24.0)$ & 0.957 \\
Hepatitis B virus (HBV) & $5(10.6)$ & $3(5.6)$ & 0.469 \\
Alcoholic liver disease & $9(19.1)$ & $5(8.7)$ & 0.268 \\
Hepatic liver failure & $11(23.4)$ & $11(19.2)$ & 0.938 \\
Primary biliary cirrhosis & $1(2,1)$ & $2(3.5)$ & 1.000 \\
Primary sclerosing cholangitis & $1(2.1)$ & $3(5.6)$ & 0.620 \\
Budd-Chiari syndrome & $2(4.2)$ & $0(0.0)$ & 0.218 \\
Autoimmune hepatitis & $3(6.3)$ & $8(14.0)$ & 0.285 \\
Cryptogenic cirrhosis & $0(0.0)$ & $4(7.0)$ & 0.120 \\
Hepatocarcinoma + HBV & $0(0.0)$ & $1(1.7)$ & 1.000 \\
Hepatocarcinoma + HCV & $2(4.2)$ & $3(5.6)$ & 1.000 \\
\hline Total & $47(99.6)$ & $53(100)$ & \\
\hline
\end{tabular}

*The chi-quadrate test. 
Table 2. Hemodynamic Data During Steps I (Initial Stages), II (Anhepatic Stages) and III (Final Stages) of 100 Patients Submitted to Orthotopic Liver Transplant Performed by Piggyback Technique with (Group A) and without (Group B) Inferior Vena Cava Cross-Clamping

\begin{tabular}{|c|c|c|c|c|}
\hline \multirow{2}{*}{ Group } & Step & MAP & CVP & HR \\
\hline \hline \multirow{3}{*}{ A } & I & $7.82 \pm 1.43$ & $9.96 \pm 3.56$ & $83.14 \pm 16.35$ \\
\cline { 2 - 5 } & II & $7.42 \pm 123^{*}$ & $8.46 \pm 4.65$ & $87.11 \pm 16.12$ \\
\cline { 2 - 5 } & III & $8.25 \pm 1.12^{*}$ & $10.40 \pm 3.72$ & $83.90 \pm 16.94 * * *$ \\
\hline \multirow{3}{*}{ B } & I & $7.81 \pm 1.25$ & $10.31 \pm 3.50$ & $92.90 \pm 14.60$ \\
\cline { 2 - 5 } & II & $7.61 \pm 1.22$ & $9.26 \pm 3.44 * *$ & $96.50 \pm 17.06^{* * *}$ \\
\hline
\end{tabular}

$\mathrm{MAP}=$ Mean arterial pressure; $\mathrm{CVP}=$ Central venous pressure; $\mathrm{HR}=$ Heart rate.

$*(\mathrm{p}<0.016)$.

$* *(\mathrm{p}=0.004)$

$* * *(\mathrm{p}<0.001)$.

non-function. A significant difference $(\mathrm{p}=0.001)$ of $1.39 \mathrm{~h}$ was recorded for the total duration of the intervention: Group $\mathrm{A}=7.13 \pm 1.90 \mathrm{~h}$ and Group B $=8.52 \pm 1.90 \mathrm{~h}$. Furthermore, a significant $(\mathrm{p}=0.016)$ higher need of PRC was observed: Group B $=5.63 \pm 4.23$ PCR and Group $\mathrm{A}=3.63 \pm$ 1.96 PCR. MAP measurements for both groups showed no significant differences during all three surgical steps (Table 2). However, when the MAP from Group A was assessed, a significant increase $(p<0.016)$ in step III was observed when compared to step II. On the other hand, the MAP for Group B showed no significant variation (Table 2). Similarly, the CVP mean for Group A and Group B had no significant difference in any of three steps. Also, no changes in CVP and HR were observed during the three operative steps in Group A. Conversely, the CVP of Group B patients showed a significant $(p=0.004)$ increase in step III, compared to step II. Additionally, HR analysis revealed a significant $(\mathrm{p}<0.001)$ increase for Group B, from step I to step III (Table 2).

When comparing mean of fluids infusion during the operation, no significant difference $(p=0.591)$ was found be- tween the Group A $(5,888 \pm 2,475 \mathrm{~mL})$ and Group B $(6,166$ $\pm 2,070 \mathrm{~mL})$. No significant difference $(p=0.535)$ was observed about the FFP administration for the two groups A and $\mathrm{B}, 3.69 \pm 1.52$ units and $4.05 \pm 1.81$ units, respectively. Conversely, differences $(p<0.050)$ were encountered when comparing mean dosis of noradrenaline administration for groups A $(4.38 \pm 1.02 \mu \mathrm{g} / \mathrm{Kg} / \mathrm{min})$ and $\mathrm{B}(3.40 \pm 1.47$ $\mu \mathrm{g} / \mathrm{Kg} / \mathrm{min})$. The mean of diuresis during the intervention showed no significant differences $(\mathrm{p}=0.396)$ when comparing the Groups A $(1,268 \pm 547 \mathrm{~mL})$ and $\mathrm{B}(1,152 \pm 549 \mathrm{~mL})$.

Serum levels of AST, ALT, TB, Cr, BUN, and INR showed no significant differences when comparing both Groups A and B, either preoperatively, or postoperatively. However, differences were encountered when comparing mean serum levels of the ALT, AST and BUN serum levels in the pre- and postoperative tests within the same Group (Table 3).

\section{DISCUSSION}

This retrospective study has evaluated the duration of intervention, the need for PRC, and the hemodynamic, bio-

Table 3. Blood Laboratory Data of 100 Patients Analyzed in the Pre and Postoperative (48 h after) Period of Orthotopic Liver Transplant Performed by Piggyback Technique with (Group A) and without (Group B) Inferior Vena Cava CrossClamping

\begin{tabular}{|c|c|c|c|c|c|c|}
\hline & \multicolumn{2}{|c|}{ Group A } & \multirow{2}{*}{$\mathbf{p}$} & \multicolumn{2}{|c|}{ Group B } & \multirow{2}{*}{$\mathbf{p}$} \\
\hline & Pre & Post & & Pre & Post & \\
\hline ALT & $262.78 \pm 71.169$ & $1117.19 \pm 165.647$ & $<0.001$ & $124.86 \pm 21.78$ & $1303.64 \pm 224.34$ & $<0.001$ \\
\hline AST & $267.58 \pm 70.33$ & $1834.76 \pm 389.28$ & $<0.001$ & $154.58 \pm 31.99$ & $1916.00 \pm 334.45$ & $<0.001$ \\
\hline BUN & $41.09 \pm 0.17$ & $82.25 \pm 0.21$ & $<0.001$ & $42.17 \pm 0.10$ & $87.41 \pm 0.19$ & $<0.001$ \\
\hline $\mathrm{Cr}$ & $1.16 \pm 0.17$ & $1.78 \pm 0.21$ & 0.06 & $1.18 \pm 0.10$ & $1.72 \pm 0.19$ & 0.11 \\
\hline INR & $2.55 \pm 0.31$ & $3.17 \pm 0.36$ & $>0.05$ & $2.40 \pm 0.34$ & $3.00 \pm 0.41$ & $>0.05$ \\
\hline
\end{tabular}

$\mathrm{ALT}=$ Alanine aminotransferase, $\mathrm{AST}=$ Aspartate aminotransferase, $\mathrm{BT}=$ Total bilirubin, $\mathrm{BUN}=\mathrm{Blood}$ urea nitrogen, $\mathrm{Cr}=\mathrm{Creatinine}, \mathrm{INR}=\mathrm{International}$ normalized rate 
chemical, and INR changes resulting from the piggyback technique with and without IVC-CC, in 100 patients undergoing OLT. No significant differences were registered concerning demographic data, ABO blood groups, etiology or severity of the hepatic disease, thus allowing a comparative analysis between the two groups. It is possible that the small number of patients evaluated in each group justified the lack of statistical significance.

The MAP calculated for surgical steps I, II, and III demonstrated no statistically significant difference between the two Groups (Table 2). One possible explanation for the MAP stability during IVC-CC would be the maintenance of the portal return of blood flow through the portal and caval systems communications with the azigous vein, which drains to the superior vena cava $[6,7]$. This explanation, however, would only apply to those patients presenting portal hypertension or previous IVC ligations, since there is insufficient time for patients with fulminant hepatic failure to establish collateral circulation $[8,9]$. However, when assessing the MAP within the same Group during the three surgical steps, it was found that in Group A, there was a MAP increase in step III compared to that of step II. It is quite possible that in order to counteract the slight but steady fall in MAP during IVC-CC (step II) fluids and vasoactive drugs were administered to maintain MAP above $7 \mathrm{mmHg}$, using this parameters in both groups. Conceivably, these procedures in conjunction with increased venous flow return after graft reperfusion, upon removal of IVC and portal vein clamping, would be responsible for the higher MAP towards the end of the surgery. It is important to highlight that although no significant differences were found, the same phenomenon was also observed for Group B. It is assumed that occluding the recipient's hepatic vein during step II by means of vascular clamping, somehow affects the IVC, and decreases its diameter. In fact, this phenomenon has already been described by Margarit et al. [10] while studying piggyback hemodynamics with partial IVC occlusion, in which a $23 \%$ reduction of the IVC flow and a $12 \%$ reduction of cardiac output were recorded. Recently, in a prospective and randomized study comparing pulmonary changes among patients undergoing OLT by the conventional technique with venovenous bypass and the piggyback technique, Isern et al. [11] registered an increase in lung infiltrate in the piggyback group. This fact corroborates the assumption that vascular clamping of the recipient's hepatic vein affects and reduces the IVC diameter, thus causing IVC hypoflow.

The measured CVP for steps I, II, and III revealed no differences between the two groups. However, when analyzing CVP values, a mean decrease of $4 \mathrm{mmHg}$ for Group A was found, from step I to step II. There is a possibility that this reduction is related to IVC-CC. On the other hand, a significant increase in CVP was found in step III as compared to step II.

The similarity of our data from Group B and the results of Isern et al. [11] evaluating the pulmonary congestion after revascularization on the conventional piggyback technique could be related to the higher reduction of the diameter of IVC during its partial clamping. We think that this clamping of IVC during the conventional piggyback is not partial, but presents hemodynamics changes just like in a cross-clamping.
The HR behavior was increasingly higher throughout the whole operative procedure in the two Groups, although only Group B patients showed a significant difference between steps I and III. It is assumed that anesthetic drug reduction could have influenced this event $[12,13]$. Both AST and ALT serum levels showed similar behaviors. In the preoperative period, no statistically significant differences were found, reflecting the homogeneity between Groups (Table 3). The excessive increase in postoperative AST and ALT serum levels for both Groups was expected, since it is related to the well-known ischemia-reperfusion injury [14].

High preoperative TB serum levels were evident (Table 3), probably as a consequence of fulminant hepatic failure, and frequently encountered in patients with cholestatic liver disease [15]. In fact, 22 patients with fulminant liver failure and 7 with cholestatic diseases were included in this series (Table 1). However, pre- and postoperative TB levels for Groups A and B revealed no significant differences, although there is an tendency to decrease during the postoperative period.

The above phenomenon was also observed in the INR statistical analysis (Table $\mathbf{3}$ ).

Although TB and INR values could reflect hepatic graft function, the second postoperative day would be too early to show a relevant recovery of the graft function.

The Cr serum level in this study did not show any significant difference between patients from Groups A and B, during pre- and postoperative periods (Table 3 ). In the midnineties, a prospective and randomized study evaluating the true outcome of renal function in relation to venovenous bypass usage, concluded that venovenous bypass did not influence either serum BUN and Cr values [16]. The only variable correlating with postoperative renal function was systemic arterial hypotension during anesthetic induction [16]. It is possible that the arterial hypotension is related to the vasoplegy so frequently encountered in patients with end-stage liver disease [17].

A later study comparing the piggyback technique without IVC-CC and the conventional procedure, which resects the IVC without the use of a venovenous bypass, revealed that a higher percentage of patients who underwent the conventional technique $-45 \%$ - developed renal failure [18]. It is worth remembering that the IVC occlusion time in the classical procedure is theoretically longer than in the piggyback with IVC-CC. In fact, restoring IVC blood flow in the classical technique requires the construction of at least three anastomosis, while in the piggyback procedure with IVC-CC only one is necessary. Another study analyzing 875 patients undergoing both the piggyback and the conventional technique with and without the use of a venous bypass, revealed no differences concerning postoperative renal function [19]. It is possible that in the present study, the behavior of Group A was similar to Group B regarding renal function, because of MAP stability, thus always maintaining values compatible with normal renal perfusion. Furthermore, the IVC-CC as determined by this technique is usually of short duration, as corroborated by Belguiti et al. [5]. Differently from serum $\mathrm{Cr}$ levels, the BUN showed a significant increase during the postoperative period in both Groups, which could be the result of the catabolic state induced by surgical trauma as well 
as high doses of corticosteroid (Table $\mathbf{3}$ ). In addition, hepatic recovery could also have contributed to the increased BUN production.

As far as time frame is concerned, the mean time for the three considered steps was $1.39 \mathrm{~h}$ less than when piggyback with IVC-CC was carried out. This is probably due to the extra time required in the conventional piggyback approach for ligation and division of the retrohepatic vessels. During the intraoperative period, Group A was seen to need less amounts of PRC than Group B. This could be due to reduced blood loss during hepatectomy with vascular clamping occlusion of the IVC retro-hepatic portion. Additionally, after suturing the vascular ostia, a test is carried out by placing clamps above and under the openings of the three hepatic veins, and rapidly loosening the vascular clamp above the renal veins. Thus, any bleeding point can easily be identified and repaired. The vascular clamping can then be closed again. This maneuver is carried out repeatedly until complete retro-hepatic IVC hemostasia has been secured. It has also been described that the conventional piggyback technique, even without IVC-CC, leads to less bleeding when compared to the classical technique with IVC resection [20-22].

Despite no significant difference regarding the amount of fluids infusion, FFP administration and diuresis during the surgery, more noradrenaline for Group A was needed.

The aim of this study was to evaluate only the early complications of the piggyback technique with and without IVC$\mathrm{CC}$, including early mortality. However, the late complications are also relevant and further prospective studies are needed.

In summary, this study shows that OLT using the piggyback technique with IVC-CC provides no major hemodynamic changes, and requires less units of blood transfusion. Nevertheless, more noradrenaline was necessary during the IVC-CC.

Furthermore, it takes less time than the piggyback technique without IVC-CC. Contrary to expectations, $48 \mathrm{~h}$ after the OLT no major changes in hepatic and renal functions were observed when compared to the non-occlusive technique. Finally, it is important to point out that this study was retrospective, evaluated a small number of patients and could had bias on the selection criteria for the groups.

Therefore, further prospective and randomized studies may be necessary to confirm these findings and to evaluate the late mortality.

\section{REFERENCES}

[1] Calne RY, Williams R. Liver transplantation in man. I. Observations on technique and organization in five cases. Br Med J 1968; 4: 535-40.

[2] Casavilla A, Gordon R, Starzel T. Techniques of the liver transplantation. In: Blumgart LH, Ed. Surgery of the liver and biliary tract. London; New York: W.B. Saunders 2001; pp. 215580.

[3] Tzakis A, Todo S, Starzl TE. Orthotopic liver transplantation with preservation of the inferior vena cava. Ann Surg 1989; 210: 64952.

[4] Ferraz AA, Sette MJ, Maia M, et al. Liver transplant for the treatment of giant hepatic hemangioma. Liver Transplant 2004; 10: 1436-7.

[5] Belghiti J, Panis Y, Sauvanet A, Gayet B, Fekete F. A new technique of side to side caval anastomosis during orthotopic hepatic transplantation without inferior vena caval occlusion. Surg Gynecol Obstet 1992; 175: 270-2

[6] Belghiti J, Ettorre GM, Durand F, et al. Feasibility and limits of caval-flow preservation during liver transplantation. Liver Transpl 2001; 7: 983-7.

[7] Lacerda CM, Sette M, Maia M, Rocha N. A ligadura prévia da veia cava inferior facilitando o transplante ortotópico do fígado. Acta Cir Bras 1995; 10: 151-5.

[8] Figueras J, Sabate A, Fabregat J, et al. Hemodynamics during the anhepatic phase in orthotopic liver transplantation with vena cava preservation: a comparative study. Transplant Proc 1993; 25: 25889.

[9] Minz M, Siwach V. Results of liver transplantation in fulminant hepatic failure. Indian J Gastroenterol 2003; 22 Suppl 2: S75-77.

[10] Margarit C, Lazaro JL, Hidalgo E, et al. Cross-clamping of the three hepatic veins in the piggyback technique is a safe and well tolerated procedure. Transpl Int 1998; 11 Suppl 1: S248-250.

[11] Isern MR, Massarollo PC, de Carvalho EM, et al. Randomized trial comparing pulmonary alterations after conventional with venovenous bypass versus piggyback liver transplantation. Liver Transpl 2004; 10: 425-33.

[12] Pichot V, Buffiere S, Gaspoz JM, et al. Wavelet transform of heart rate variability to assess autonomic nervous system activity does not predict arousal from general anesthesia. Can J Anaesth 2001; 48: 859-63

[13] Kay B, Nolan D, Mayall R, Healy TE. The effect of sufentanil on the cardiovascular responses to tracheal intubation. Anaesthesia 1987; 42: 382-6.

[14] Lopez M, Gamez M, Vazquez J, et al. Biochemical indicators of primary graft dysfunction in experimental orthotopic liver transplantation. Cir Pediatr 1995; 8: 63-7.

[15] Sherlock S, Dooley J: Assessment of the liver function. In: Sherlock S, Dooley J, Eds. Diseases of the liver and biliary system. $11^{\text {th }}$ ed. London: Blackwell Publishing, 2002; pp. 19-36.

[16] Grande L, Rimola A, Cugat E, et al. Effect of venovenous bypass on perioperative renal function in liver transplantation: results of a randomized, controlled trial. Hepatology 1996; 23: 1418-28.

[17] Ponnudurai RN, Koneru B, Akhtar SA, et al. Vasopressor administration during liver transplant surgery and its effect on endotracheal reintubation rate in the postoperative period: a prospective, randomized, double-blind, placebo-controlled trial. Clin Ther 2005; 27: 192-8.

[18] Nemes B, Kobori L, Fehervari I, et al. Comparison of the results of conventional, crossclamp and piggyback technique in liver transplantation. Magy Seb 2005; 58: 155-61.

[19] Moreno-Gonzalez E, Meneu-Diaz JG, Fundora Y, et al. Advantages of the piggy back technique on intraoperative transfusion, fluid compsumption, and vasoactive drugs requirements in liver transplantation: a comparative study. Transplant Proc 2003; 35: 1918-9.

[20] Gonzalez FX, Garcia-Valdecasas JC, Grande L, et al. Vena cava vascular reconstruction during orthotopic liver transplantation: a comparative study. Liver Transpl Surg 1998; 4: 133-40.

[21] Lerut JP, Molle G, Donataccio M, et al. Cavocaval liver transplantation without venovenous bypass and without temporary portocaval shunting: the ideal technique for adult liver grafting? Transplant Int 1997; 10: 171-9.

[22] Miyamoto S, Polak WG, Geuken E, et al. Liver transplantation with preservation of the inferior vena cava. A comparison of conventional and piggyback techniques in adults. Clin Transplant 2004; 18: 686-93. 